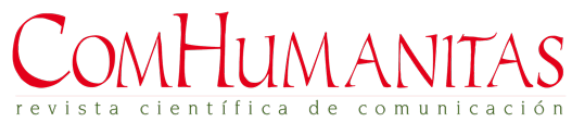

Revista ComHumanitas, ISSN: 1390-776X

Vol 10, núm. 2 (2019), Mayo-agosto 2019

DOI: https://doi.org/10.31207/rch.v10i2.202

\title{
El Femvertising como estrategia de Comunicación y su impacto en mujeres entre 25 y 35 años en la ciudad de Quito - Ecuador
}

\section{Femvertising as a strategy of Communication and its impact on women between 25 and 35 years old in the city of Quito - Ecuador}

\section{A propaganda publicitária como estratégia de comunicação e seu impacto em mulheres entre 25 e 35 anos na cidade de Quito - Equador}

\author{
Marco López Paredes \\ Pontificia Universidad Católica del Ecuador ${ }^{1}$ \\ mvlopez@puce.edu.ec \\ María Fernanda García ${ }^{2}$ \\ Pontificia Universidad Católica del Ecuador \\ fergh95@gmail.com
}

Fecha de recepción: 5 de abril de 2019

Fecha de recepción evaluador: 20 de abril de 2019

Fecha de recepción corrección: 3 de mayo de 2019

\footnotetext{
${ }^{1}$ Marco López Paredes, Ph.D en Comunicación, Post.Doc. Experto en redes sociales, estrategias de marca y redes sociales. Investigador del fenómeno de la comunicación. Revisor científico Revista Comunicar. https://orcid.org/0000-0001-7853-5564

${ }^{2}$ María Fernanda García, Comunicadora Organizacional del a Pontificia Universidad Católica del Ecuador con experiencia en Marketing Digital, Relaciones Públicas y Periodismo. Inició su carrera profesional como pasante en el Diario El Comercio y en Ekos (Revista Estilo). https://orcid.org/0000-0002-2205-4312
} 


\title{
Resumen
}

Este trabajo busca identificar la apreciación de las mujeres frente al uso de estrategias profeminismo en la industria publicitaria con una nueva estrategia de comunicación publicitaria: el femvertising. Su principal objetivo es eliminar los estereotipos peyorativos o cosificativos de la mujer, que durante décadas han sido replicados y legitimados en la publicidad, esto desde un mensaje positivo que empodere a las mujeres, invitándolas a explorar nuevos roles y a elevar su autoestima. La investigación toma como ejemplo al Ecuador y su capital, que se caracterizan por presentar estadísticas altas de violencia de género y machismo la información y datos fueron obtenidos a través de un grupo focal, en los que se analizaron los estímulos generados por tres spots publicitarios alineados a la tendencia del femvertising: "This is Us" de Nike, "Legado" de Dove y "Nosotras cambiamos el Bullying por Loving" de Nosotras Ecuador.

Palabras clave: femvertising, feminismo, comportamiento del consumidor, actitudes del consumidor, publicidad, publicidad social.

\begin{abstract}
This paper seeks to identify the appreciation of women against the use of pro-feminist strategies in the advertising industry with a new advertising communication strategy: femvertising. Its main objective is to eliminate the pejorative or materialistic stereotypes of women, which for decades have been replicated and legitimized in advertising, this from a positive message that empowers women, inviting them to explore new roles and raise their self-esteem. The research takes as an example Ecuador and its capital, which are characterized by presenting high statistics of gender violence and sexism, information and data were obtained through a focus group, in which the stimuli generated by three advertising spots aligned were analyzed. to the trend of femvertising: "This is Us" by Nike, "Legacy" by Dove and "We change Bullying by Loving" from Nosotras Ecuador.
\end{abstract}

Keywords: "fem-vertising", feminism, consumer behaviour, consumer attitudes, advertising, social advertising.

\section{Resumo}

Este artigo procura identificar a valorização das mulheres contra o uso de estratégias prófeministas no setor publicitário, com uma nova estratégia de comunicação publicitária: femvertising. Seu principal objetivo é eliminar os estereótipos pejorativos ou materialistas das mulheres, que há décadas são replicados e legitimados na publicidade, isso a partir de uma mensagem positiva que capacita as mulheres, convidando-as a explorar novos papéis e elevar sua auto-estima. A pesquisa toma como exemplo o Equador e sua capital, que se caracterizam por apresentar altas estatísticas de violência de gênero e sexismo, informações e dados foram obtidos por meio de um grupo focal, no qual foram analisados os estímulos gerados por três anúncios alinhados. à tendência do femvertising: "This is Us", da Nike, "Legacy", de Dove, e "We change Bullying by Loving", da Nosotras Ecuador. 
Palavras-chave: feminismo, feminismo, comportamento do consumidor, atitudes do consumidor, publicidade, publicidade social.

\section{Introducción}

Ahora más que nunca vemos como el modelo de comunicación basado en la realimentación se hace efectivo. La interacción y el cambio de roles entre emisor y perceptor se expande y está presente en todo tipo de institución: familiar, civil, gubernamental o mercantil. Sobre todo, en esta última, en donde las marcas le han dedicado grandes inversiones en investigación y producción de piezas que logren captar la atención de sus consumidores en medio de un océano de información, en el que la mayoría pasa desapercibida.

Recientemente, las marcas junto con sus agencias de publicidad han optado por camuflar sus intenciones de venta con causas apegadas a los intereses de sus consumidores. De tal manera, que, al ver sus contenidos, sea imposible no captar su atención. De ahí que la publicidad haya puesto los ojos en una de las demandas sociales más controversiales en los últimos tiempos: el feminismo. Pese a que ha existido desde el siglo XIX, no es hasta los años 70 que empieza a convertirse en un movimiento social activo y global. Esto gracias a su repercusión en internet y, principalmente, en redes sociales. Ahora quienes defienden el feminismo, poden alzar su voz y exigir una publicidad más realista y menos limitada por los roles de género caducos y tradicionales, en los que se mostraba a la mujer como sumisa, hogareña, maternal o sexual.

Es en este contexto, hace 4 años se decidió apostar por una nueva manera de hacer publicidad; en la que se reemplacen los arquetipos lascivos y machitas por mensajes y contenido que empodere a la mujer. Esta tendencia es conocida como femvertising, una estrategia de comunicación publicitaria desarrollada y popularizada en Estados Unidos por la agencia She Knows Media. Dicho concepto integra dos términos anglosajones: female (femenino) y advertising (publicidad) por lo que:

"busca mediante la comunicación y la publicidad, mostrar a la mujer bajo una realidad que se contraponga a los paradigmas patriarcales, siendo ahora la portavoz de igualdad y empoderamiento.

Es una publicidad igualitaria que suscita el empoderamiento desde todas las vertientes sociales en que se desenvuelven las mujeres." (Ojeda, 2016, p.2)

Esta propuesta publicitaria ha adquirido gran popularidad y réplica en países como Estados Unidos y Europa; sin embargo, en Latinoamérica se presenta un panorama incipiente, en donde la producción de spots con este enfoque aún no logra captar su esencia y se sigue cayendo en los esquemas tradicionales de representación de la mujer. Lo mismo sucede en Ecuador, en donde no se han presentado mayores cambios en la construcción de la imagen de la mujer en la publicidad. Por esto el estudio muestra cómo se ha construido la representación de la mujer en la publicidad, sus principales estereotipos y su evolución 
hasta llegar al femvertising. Finalmente, se expone la metodología de investigación y el análisis de los resultados.

\section{Marco Teórico}

\section{Construcción de la identidad social y colectiva}

Para determinar la influencia de la publicidad y la comunicación publicitaria en la construcción de los estereotipos es necesario conocer: qué aspectos las constituyen, su impacto a nivel individual y la manera de transformarlos o afianzarlos. Para ello es importante comprender que las directrices sociales no actúan de forma aislada, sino que son parte de una categoría mayor que regula el comportamiento, la actitud y la percepción de los individuos; es decir, la identidad social.

El hablar de identidad social nos remite indispensablemente a la Teoría de Identidad Social o TIS propuesta por Tajfel (1984), en la que señala que los individuos edifican su autoconcepto en base al "conocimiento de su pertenencia a ciertos grupos sociales y a la significación emocional y evaluativa que resulta de esta pertenencia” (p.150). Es decir, que los seres humanos construyen su identidad a partir de las características que se le han atribuido al grupo social al que pertenece (endogrupo) y a las concepciones que son el resultado de comparaciones frente a otros con los que existe algún tipo de relación (exogrupo).

Asimismo, una identidad negativa puede afectar las relaciones de interdependencia en temas evolutivos y a nivel socioeconómico y de producción; así lo asegura Bourhis, Gagnon, \& Moïse (1996) al mencionar que "las desigualdades de poder y de status entre los grupos sociales acarrean inevitablemente desigualdades en la distribución de recursos materiales y simbólicos" (p. 157). Esto se debe a que las construcciones ideológicas ejercen su influencia en la percepción de los grupos haciendo que el grupo social aplaque sus intenciones de reivindicación.

Los mecanismos de deformación cognitiva, habitualmente elaborados por el grupo dominante, pueden llevar a los miembros de los grupos desfavorecidos a considerar su situación como equitativa y legítima. Esta legitimación de la situación disminuye el deseo de los grupos desfavorecidos de emprender acciones colectivas necesarias para mejorar su situación. (Cruz Souza, 2011, p.51).

Para que exista cualquiera de los dos escenarios, las valoraciones del endogrupo deben cumplir con dos condiciones simbólicas básicas: la estabilidad y la legitimidad. La primera tiene un carácter histórico porque son rasgos transmitidos de generación en generación y la segunda es determinada por la familia, las instituciones educativas y los medios masivos. Siendo estos últimos quienes, en las tres décadas recientes, "se han convertido en grandes legitimadores y promotores de identidades sociales, porque construyen la imagen de las personas y las transmiten a gran escala para que el resto se refleje en ellas" (Fair, 2011). 
Con esto presente, podemos asegurar que, si bien los medios de masas no son los únicos mediadores en el proceso de conciliación de imaginarios, sí son quienes ejercen un papel protagonista en la legitimación y consolidación de actitudes y comportamientos sociales. Siendo uno de los más controversiales la publicidad, "porque su discurso posee una gran fuerza de persuasión, que influye en la transmisión de valores y creencias" (Abuín, 2009, p. 8) y sus narraciones ficcionales se erigen en "construcciones ideológicas, que marcan formas de pensar y modos de conducta" (J. García, 2015).

Al respecto, (García Canclini, 2001) señala que la mediatización social evocada por la publicidad comercial es un "juego de ecos", ya que las significaciones se construyen en base a experiencias de larga duración; es decir, lo que se ve en medios masivos se corrobora en la "calle" o en situaciones cotidianas, y viceversa.

\section{Estereotipos de la mujer en la publicidad}

En su mayoría la asignación de estereotipos y roles se realizaban según el género entiéndase a este como un constructo asignado por un contexto cultural y social (Catterall, Maclaran, \& Stevens, 1997) - atribuyendo determinadas personalidades y rasgos para mujeres y hombres. "A las mujeres se las retraba como sensibles, cálidas, dependientes y orientadas a la gente, en tanto que a los hombres se les veía como dominantes, independientes, orientados hacia el trabajo y agresivos" (Williams y Best, citado en Miero, 2015). Pese a que estos rasgos afectaban a ambos miembros de la sociedad, cuantitativa y cualitativamente, las mujeres fueron las más afectadas, porque a esto se sumaba que su imagen respondía a cánones de belleza perfectos e inalcanzables con el fin de utilizarlos como un elemento provocativo que incitaba al consumo y apelaba a los instintos más básicos del ser humano.

Asimismo, era completamente normal que la mujer apareciese desempeñando únicamente el papel de ama de casa, madre y esposa, dependiente del varón y con una escasa o nula participación en los asuntos de carácter social" (Abuín, 2009, 14).

Para los años ochenta vemos un panorama relativamente diferente; el porcentaje de spots con roles tradicionales disminuye y comienzan a aparecer spots en los que las mujeres se muestran como protagonistas y adquieren mayor independencia y libertad de decisión. Sin embargo, no es hasta finales de los 90 que este tema se consolida y las mujeres son vistas desde una perspectiva diferente a la habitual. Sánchez Aranda (2003), lo expone de la siguiente manera:

"De un plano de inferioridad con respecto al hombre se ha pasado a uno de igualdad $\mathrm{y}$, en ocasiones, de superioridad. Hombre y mujer ya no aparecen como realidades antagónicas, sino como complementarias. Y no se puede tampoco dejar de destacar cómo se ha acentuado su carácter decidido con unos comportamientos prepotentes, que suponen una subversión y asunción del tan denostado "machismo" (protagonizado ahora por personas de sexo femenino) de hace décadas" (Sánchez Aranda, 2003, p. 73).

También puede ser útil el postulado de Williams y Best (1982) para hacernos entender que el cambio no se produce de manera natural y consensuada, debido a que esta 
nueva imagen de la mujer no encaja con el estereotipo que típicamente se asignaba a las mujeres. Además, como señala Morales (1995, citado en Gonzales, 1999), se sigue viendo a la mujer con características diferentes a las de los hombres; por ello a la mujer centrada en su trabajo e independiente, se la ve como "masculina", dando a entender que lo que antes sirvió para justificar la asignación diferencial de roles, ahora sirve para frenar su cambio.

Para conocer el contexto de la representación de la mujer en la publicidad y su evolución global, Miero (2015) propone un cuadro en el que se sintetizan estos aspectos:

Tabla 1 Síntesis de la evolución histórica de la representación de la mujer en la publicidad

\begin{tabular}{|c|c|c|}
\hline Contexto & Conclusiones & Autores \\
\hline 1958-1979 & $\begin{array}{l}\text { Mayor número de hombres que mujeres en } \\
\text { los anuncios. } \\
\text { Representación muy estereotipada de } \\
\text { mujer y hombre. } \\
\text { Infrarrepresentación de la mujer en el } \\
\text { ámbito profesional, mujer representada } \\
\text { como ama de casa. } \\
\text { Mujer como elemento decorativo en } \\
\text { anuncios excluida de la toma de } \\
\text { decisiones. } \\
\text { Se prevé una representación menos } \\
\text { estereotipada en la próxima década. }\end{array}$ & $\begin{array}{l}\text { Courtney y Lockeretz (1971) } \\
\text { Dominick y Rauch (1972) } \\
\text { McArthur y Resko (1975) } \\
\text { Belkaoui y Belkaoui (1976) } \\
\text { Lundstrom y Sciglimpaglia (1977) } \\
\text { Sciglimpaglia et al. (1979) }\end{array}$ \\
\hline $\begin{array}{c}\text { 1958-1989 } \\
\text { EEUU } \\
\text { Reino Unido } \\
\text { Italia }\end{array}$ & $\begin{array}{l}\text { Uso de estereotipos, pero presencia de una } \\
\text { nueva tendencia de "súper-mujer". } \\
\text { Representación de la mujer mucho más } \\
\text { estrecha y tradicional que la del hombre. } \\
\text { Dificultad de la publicidad para seguir el } \\
\text { paso de los cambios sociales. } \\
\text { Representación de la mujer ceñida a su } \\
\text { atractivo físico, su rol de madre, esposa y } \\
\text { ama de casa. Interpretadas como usuarias } \\
\text { de producto, pero sobre todo productos de } \\
\text { uso doméstico. } \\
\text { No se cumple con las previsiones } \\
\text { anteriores, aunque empieza a haber un } \\
\text { incremento de la imagen de la mujer como } \\
\text { profesional. }\end{array}$ & $\begin{array}{l}\text { Manstead y McCulloch (1981) } \\
\text { Courtney y Whipple (1985) } \\
\text { Coughlin y O'Connor (1985) } \\
\text { Whipple y Courtney (1985) }\end{array}$ \\
\hline
\end{tabular}




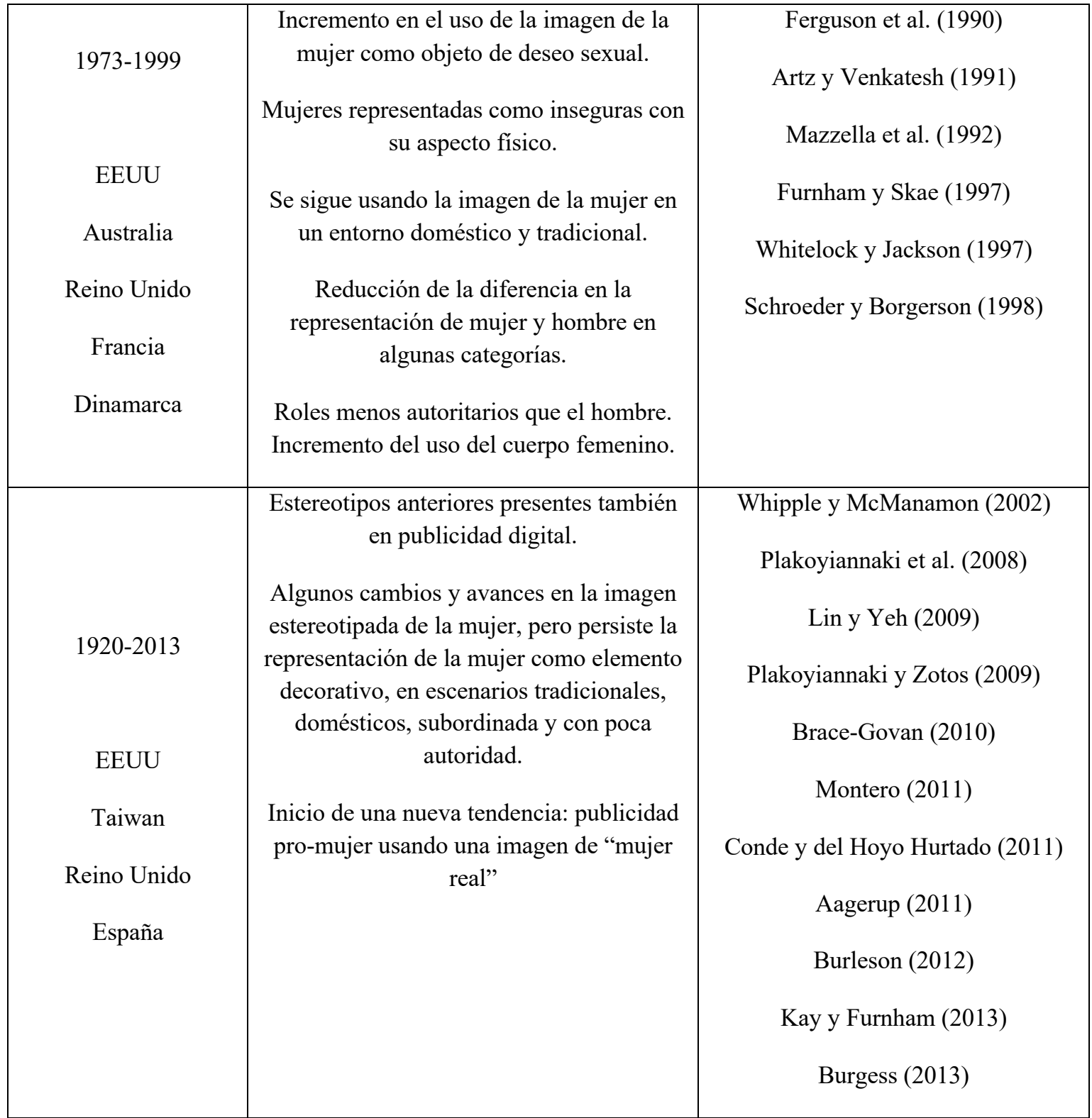

Fuente: (Miero, 2015, p. 13).

Como vemos, aunque la imagen de la mujer en la publicidad ha ido evolucionando, aún quedan los rezagos del discurso tradicional y de los estereotipos arcaicos, principalmente, en el tema estético, debido a que, en la mayoría de los spots solo se ha cambiado el contexto en el que las mujeres se desarrollan, mientras que la forma de representarlas sigue apegada a los típicos cánones de belleza.

\section{El Femvertising y la publicidad}

La estrecha relación entre los consumidores y las marcas, los múltiples estudios que corroboran el impacto de la publicidad en la percepción colectiva y los informes que aseguran que el compromiso con la igualdad de género en las empresas podría añadir al PIB mundial unos 12.000 millones de dólares hasta 2025 (Canto, 2017) han popularizado una nueva tendencia publicitaria: el femvertising. Este término proviene de dos términos anglosajones: female (femenino) y advertising (publicidad) y su principal objetivo es 
sustituir los mensajes sexistas por un discurso que empodere a la mujer. Alrededor de esta tendencia existen varias definiciones; las principales, a continuación:

El femvertising busca mostrar a la mujer bajo una realidad que se contraponga a los paradigmas patriarcales, siendo ahora la portavoz de igualdad y empoderamiento. Una publicidad igualitaria que suscita el empoderamiento desde todas las vertientes sociales en que se desenvuelven las mujeres. (Ojeda, 2016, p.2).

El femvertising se puede definir como "mensajería pro-femenina dentro de la publicidad". Es contenido que tiene un mensaje feminista y una representación no estereotipada de las mujeres. Es positivo, fortalecedor y poderoso. (Pérez \& Almanzor, 2017).

No obstante, la definición oficial es la acuñada por SheKnows Media, la plataforma multimedia que ideó y popularizó esta tendencia en los Estados Unidos. Ellos señalan que el femvertising es toda "publicidad que emplea talento, mensajes e imágenes a favor de las mujeres para empoderar a mujeres y niñas" (SheKnows Media, 2014).

Dicha tendencia surgió en el 2014 y hasta el momento ha logrado captar la atención de marcas importantes en el mercado internacional, como: Dove, Nike, Always, Covergirl y Pantene (Wojcicki, 2016). Tal fue su repercusión que en el 2015 se llevó a cabo la primera edición de los She Knows Media Awards; una convocatoria para premiar la publicidad con este enfoque, siendo Dove la más galardonada y consolidándose como líder de esta tendencia

La campaña que catapultó a Dove fue Real Beauty, lanzada en el 2004, que tenía como principal objetivo incentivar a las mujeres a cuestionar los ideales de belleza y a sentirse seguras con su cuerpo, a través de mensajes que demostrarán que las mujeres son hermosas, sin importar su tamaño o color. Durante los siguientes años, la marca se convirtió en embajadora de mensajes alineados a su visión: "hacer de la belleza una fuente de confianza y no de ansiedad". (Dove, 2018).

En este sentido, una de las principales condiciones para la efectividad del femvertising es alinear toda la comunicación y acciones corporativas en función del empoderamiento y reivindicación de la mujer. En cuanto a la generación de la estrategia publicitaria y la creación del spot en sí, Becker-Herby (2016) propone varias premisas que resumen la esencia del femvertising:

1. Utilización de talento femenino diverso.

2. Mensajes intrínsecamente pro-feministas

3. Perspectiva desafiante de los estereotipos y roles de género

4. Mínima o nula participación masculina.

5. Mujeres retratadas de forma auténtica.

(Becker-Herby, 2016). 
El primer pilar del femvertising es la diversidad del talento femenino, es decir que las mujeres que aparezcan en los spots deben ser de contexturas, etnias y rasgos diversos, de tal manera que se valore y se reconozca la autenticidad y diversidad de la mujer. Respecto al siguiente pilar, los mensajes clave de esta tendencia deben destacar el rol de la mujer en la sociedad y resaltar su capacidad para desenvolverse con éxito en cualquier rol social. De igual manera, se concentra en reemplazar los viejos estereotipos y mostrar una imagen renovada y fresca de la mujer. En la mayoría de casos, se la retrata de forma protagónica, de tal manera que se excluye al género masculino. La sinergia de estas características ayuda a que las consumidoras desarrollen un sentido de identificación y aprobación con los spots presentados y, además, contribuye al cambio de la imagen de la mujer en la publicidad y a legitimar y normalizar los nuevos logros y retos de la mujer en la sociedad.

Para ejemplificar el femvertising mencionaremos el siguiente caso entre otros desarrollados:

\section{Barbie - \#YOUCANBEANYTHING}

La campaña de Barbie se lanzó en el 2015 bajo el concepto Imagine the possibilities y ha sido reproducida más de 23 millones de veces en YouTube. El spot muestra que las mujeres pueden desempeñar cualquier tipo de oficios, sin importar lo demandante o segmentados que estos sean. Esta pieza de femvertising no solo tiene un mensaje que empodera a la mujer, sino que demuestra que la elección de una carrera no debe estar regida por roles de género.

\section{Marco Metodológico}

Este estudio se llevó a cabo de forma empírica, bajo una modalidad de investigación cualitativa. Las investigaciones con este enfoque se "centran en la comprensión de una realidad considerada desde sus aspectos particulares como fruto de un proceso histórico de construcción y vista a partir de la lógica y el sentir de sus protagonistas, es decir desde una perspectiva interna (subjetiva)" (Pérez, 2001). De tal manera que esta técnica permitirá recolectar detalles valiosos para comprender e interpretar el comportamiento de las personas y su percepción al recibir ciertos estímulos comunicativos, mismo que necesitan una explicación y análisis más allá de las cifras o porcentajes.

\section{Técnicas e instrumentos de investigación}

Para cumplir con los objetivos planteados en el presente estudio, se realizó un grupo focal junto con un cuestionario de actitudes y una matriz de análisis de contenido de los spots publicitarios; esto con el fin de proveer la información necesaria y suficiente para validar y complementar los resultados de la investigación. Tanto el grupo focal como el cuestionario fueron aplicados a diez mujeres de 25 a 35 años del Centro Norte de Quito, el porqué de la selección de dichas variables sociodemográficas serán expuestas en los próximos apartados. 
Con la ejecución de un grupo focal, se buscó conocer las reacciones de las participantes frente al femvertising, para lo cual se elaboró una serie de preguntas direccionadas a recabar tal información y extraer otros datos que aporten a mejorar la compresión de dichos estímulos. Además, se realizó un proceso estricto de selección de anuncios de publicidad basados en la tendencia del femvertising, este proceso estuvo mediado por parámetros importantes para enriquecer el estudio, mismos que serán explicados más adelante.

\section{Grupo Focal}

Se seleccionó al grupo focal como técnica de investigación, debido a que permite medir las reacciones, percepciones y actitudes de los participantes, pudiendo entender su proceso de razonamiento ante la problemática expuesta. [Krueger y Casey, 2000 (citado en Mieiro, 2015)]. Este atributo se adapta perfectamente a lo que el presente estudio requiere para obtener datos que recreen el posible panorama y efectividad del femvertising en el país.

Otra característica valiosa de esta técnica es que congrega a un número reducido de participantes, de 4 a 12 aproximadamente, lo que genera un ambiente que invita a la reflexión y al debate, permitiendo que emerjan nuevas opiniones alrededor del tema planteado inicialmente. [Krueger y Casey, 2000 (citado en Mieiro, 2015)]

Tabla 2 Instrumento para el grupo focal

\section{GUIA DE GRUPO FOCAL}

\section{Instrucciones:}

Durante esta sesión, se proyectarán varios spots publicitarios, por favor, véanlos naturalmente, como si los estuvieran observando en televisión o por redes sociales.

El objetivo de estos spots es cambiar los típicos mensajes publicitarios, enfocados únicamente en vender el producto. Fíjense también en la representación de la mujer. Al final, se realizará una ronda de preguntas respecto a su opinión frente a lo observado.

\begin{tabular}{|c|c|c|}
\hline Tema & Objetivo & Pregunta \\
\hline \multirow[b]{2}{*}{ Percepción } & \multirow{2}{*}{$\begin{array}{l}\text { Conocer las reacciones de } \\
\text { las consumidoras frente al } \\
\text { femvertising }\end{array}$} & ¿Qué opinas sobre el spot? \\
\hline & & $\begin{array}{l}\text { ¿Te sientes identificada con } \\
\text { él? } \mathrm{Si} / \mathrm{No} \text { ¿Por qué? }\end{array}$ \\
\hline \multirow{4}{*}{$\begin{array}{l}\text { Influencia del } \\
\text { mensaje }\end{array}$} & \multirow{4}{*}{$\begin{array}{l}\text { Identificar la influencia de } \\
\text { los mensajes publicitarios } \\
\text { presentados en los spots de } \\
\text { femvertising }\end{array}$} & $\begin{array}{l}\text { ¿Cuál crees que es la } \\
\text { finalidad de la marca al hacer este }\end{array}$ \\
\hline & & spot? \\
\hline & & $\begin{array}{l}\text { Del spot, ¿con qué palabra o } \\
\text { frase te quedas? }\end{array}$ \\
\hline & & $\begin{array}{c}\text { ¿Qué tienen en común estos } 3 \\
\text { spots? }\end{array}$ \\
\hline
\end{tabular}




\begin{tabular}{|c|c|c|}
\hline $\begin{array}{l}\text { Representación } \\
\text { de la mujer }\end{array}$ & $\begin{array}{l}\text { Determinar cómo perciben } \\
\text { la representación de las mujeres en el } \\
\text { spot }\end{array}$ & $\begin{array}{l}\text { Para ti, ¿cómo se representa a } \\
\text { la mujer en esta publicidad? }\end{array}$ \\
\hline \multirow{3}{*}{ Efectividad } & \multirow{3}{*}{$\begin{array}{l}\text { Identificar los elementos } \\
\text { que determinar la efectividad o no } \\
\text { efectividad del femvertising }\end{array}$} & $\begin{array}{c}\text { ¿El spot te motiva a la } \\
\text { compra de la marca? Si, No, ¿Por } \\
\text { qué? }\end{array}$ \\
\hline & & $\begin{array}{l}\text { Si hubieses participado en la } \\
\text { construcción de este spot ¿Qué le } \\
\text { hubieses quitado o agregado? }\end{array}$ \\
\hline & & $\begin{array}{l}\text { Compartirías alguno de estos } \\
\text { tres spots en redes sociales. Si, No, } \\
\text { ¿Cuál? }\end{array}$ \\
\hline $\begin{array}{l}\text { Influencia del } \\
\text { feminismo }\end{array}$ & $\begin{array}{l}\text { Determinar si el feminismo } \\
\text { media la percepción de las } \\
\text { consumidoras frente al femvertising }\end{array}$ & $\begin{array}{l}\text { Hay personas que catalogan a } \\
\text { esta publicidad como feminista. ¿Tú } \\
\text { qué opinas al respecto? ¿Por qué? }\end{array}$ \\
\hline
\end{tabular}

Fuente: Elaboración de los autores

\section{Selección de los anuncios}

Para la selección de los spots que serían analizados en el grupo focal, se utilizó el buscador de Google, YouTube y Vimeo para detectar marcas que hayan incursionado en esta tendencia; no obstante, la mayoría de las campañas eran de otros países y estaban en otros idiomas. Por lo que los parámetros de selección fueron en base a los pilares del femvertising propuestos por Becker-Herby (2016)

1. Utilización de talento femenino diverso

2. Mensajes intrínsecamente profeministas

3. Perspectiva desafiante de los estereotipos y roles de género

4. Minimiza o anula la participación masculina

5. Intenta retratar mujeres de forma auténtica

Los spots seleccionados son: Legado de Dove; This is Us de Nike y Nosotras cambiamos el Bullying por Loving de Nosotras Ecuador. Los tres cumplen con las características propias del femvertising y cumplen con otras condiciones importantes para diversificar las opiniones en el grupo focal. Por ello, se consideró que entre los seleccionados debe estar un spot emotivo, uno energético y uno tropicalizado, es decir, creado exclusivamente para Ecuador.

El primero fue seleccionado porque pertenece a la marca ganadora en varias ocasiones de los Femvertising Awards, consagrándose como líder de esta tendencia. Pese a que la voz en off de Legado es en inglés, la marca lanzó esta campaña para Latinoamérica, 
agregándole subtítulos en español. Además, cumple con el requisito de ser emocional, necesario para dar un equilibrio entre los tres spots.

El siguiente spot es This is Us de Nike, esta pieza fue construida en turco y tiene subtítulos en inglés; sin embargo, se la incluyó en el presente análisis por la calidad de escenas y concreción en el mensaje. Además, fue necesario, realizar una traducción propia y editar el video para agregarle los subtítulos en español. Este spot cumple con la cuota de energético.

Finalmente, se eligió a Nosotras cambiamos el Bullying por Loving debido a que Nosotras es una marca más cercana a las participantes y, sobre todo, tiene elementos propios del Ecuador debido a que es una producción nacional. De hecho, esta es la primera marca en el país que realiza una campaña alineada a esta tendencia. Aunque los actantes de este spot son adolescentes, el mensaje es universal. Esta pieza es la tropicalizada.

\section{Matriz de análisis de los anuncios}

Uno de los objetivos de la investigación es identificar qué elementos determinan la efectividad de los spots publicitarios bajo la tendencia del femvertising, por esta razón es clave realizar un análisis descriptivo de cada una de las piezas publicitarias seleccionadas para la proyección en el grupo focal. Además, este análisis permitirá corroborar con fundamentos las opiniones dadas por las participantes y descubrir qué factores de composición pudieron influir en su respuesta. Con el fin de conseguir mejores resultados, se hizo una investigación del contexto de cada spot y se los dividió en escenas para examinar cada una con el mismo proceso analítico. 
Tabla 3 Modelo de la matriz de análisis de los anuncios publicitarios

\begin{tabular}{|c|c|c|c|c|c|c|c|c|c|}
\hline \multicolumn{10}{|c|}{ Descripción del anuncio publicitario } \\
\hline \multicolumn{10}{|c|}{$\begin{array}{l}\text { En esta sección se contextualiza al spot y se detalla la narrativa en la que está sustentado. También se coloca datos relevantes de la composición audiovisual, semántica } \\
\text { o discursiva, de tal manera que se provea de una introducción basta para la comprensión del análisis secuencial. }\end{array}$} \\
\hline Secuencia & Duración & Descripción & $\begin{array}{l}\text { Framing } \\
\text { Visual }\end{array}$ & $\begin{array}{l}\text { Framing } \\
\text { Verbal }\end{array}$ & $\begin{array}{l}\text { Framing } \\
\text { Aureal }\end{array}$ & $\begin{array}{l}\text { Figura } \\
\text { Retórica }\end{array}$ & Connotación & $\begin{array}{l}\text { Connotación del } \\
\text { color }\end{array}$ & $\begin{array}{l}\text { Sentido de } \\
\text { la escena }\end{array}$ \\
\hline $\begin{array}{c}\text { El spot debe } \\
\text { ser dividido en } \\
\text { secuencias; es } \\
\text { decir, en } \\
\text { conjuntos de } \\
\text { planos o } \\
\text { escenas que } \\
\text { ordenados } \\
\text { crean una } \\
\text { línea } \\
\text { argumental. } \\
\text { En literatura, } \\
\text { es análogo a } \\
\text { un capítulo. }\end{array}$ & $\begin{array}{c}\text { Se debe } \\
\text { colocar el } \\
\text { segundo } \\
\text { exacto de } \\
\text { inicio y fin } \\
\text { de la escena. }\end{array}$ & $\begin{array}{c}\text { Cada secuencia } \\
\text { debe ser descrita } \\
\text { de forma } \\
\text { detallada, } \\
\text { prestando } \\
\text { atención a los } \\
\text { elementos más } \\
\text { relevantes. }\end{array}$ & $\begin{array}{c}\text { Representa } \\
\text { los } \\
\text { elementos } \\
\text { visuales que } \\
\text { compone el } \\
\text { spot. Tales } \\
\text { como: } \\
\text { logos, texto, } \\
\text { locación, } \\
\text { actantes, } \\
\text { etc. }\end{array}$ & $\begin{array}{l}\text { Es el guion } \\
\text { que } \\
\text { acompaña } \\
\text { al spot. Este } \\
\text { puede ser } \\
\text { expuesto } \\
\text { mediante el } \\
\text { discurso de } \\
\text { un actante o } \\
\text { por voz en } \\
\text { off }\end{array}$ & $\begin{array}{c}\text { Se refiere a } \\
\text { los recursos } \\
\text { que } \\
\text { acompañan } \\
\text { la composi } \\
\text { ción visual. } \\
\text { Tales como: } \\
\text { la música o } \\
\text { sonidos de } \\
\text { ambiente. }\end{array}$ & $\begin{array}{c}\text { Insta a } \\
\text { señalar los } \\
\text { recursos } \\
\text { estéticos } \\
\text { que utiliza } \\
\text { la escena } \\
\text { para } \\
\text { trasmitir un } \\
\text { mensaje. }\end{array}$ & $\begin{array}{c}\text { Se develan } \\
\text { aquellos } \\
\text { elementos que } \\
\text { aportan un } \\
\text { sentido semántico } \\
\text { a la composición } \\
\text { del spot y se } \\
\text { presenta una } \\
\text { interpretación } \\
\text { más elaborada de } \\
\text { los expuesto en la } \\
\text { descripción. }\end{array}$ & $\begin{array}{l}\text { La composición } \\
\text { visual y los tonos } \\
\text { de las escenas } \\
\text { aportan una } \\
\text { ambientación } \\
\text { apelativa a los } \\
\text { sentidos. En este } \\
\text { apartado se debe } \\
\text { describir las } \\
\text { intenciones que } \\
\text { cada escena } \\
\text { manifiesta en el } \\
\text { color. }\end{array}$ & $\begin{array}{c}\text { Es una } \\
\text { síntesis de } \\
\text { todos los } \\
\text { aspectos } \\
\text { analiza- } \\
\text { dos y una } \\
\text { hipótesis } \\
\text { del porqué } \\
\text { de la } \\
\text { escena.. }\end{array}$ \\
\hline
\end{tabular}

Fuente: Elaboración propia 


\section{Resultados}

Con relación a las afirmaciones acerca de la imagen de la mujer en la publicidad dio como resultado que la mayoría cuestiona la forma en que los anuncios publicitarios retratan a la mujer, mientras que una minoría se muestra más tolerante frente a este hecho. El hecho de que la mayoría de las participantes tiendan a rechazar el contenido que presenta a la mujer de forma sexista, da indicios de que serán más propensas a sentir identificación y de mostrarse más receptivas a los contenidos alineados al femvertising.

Tabla 4 Resultados del cuestionario de actitudes hacia la publicidad en general

ACTITUDES HACIA LA PUBLICIDAD

\begin{tabular}{c|ccccccccc}
\hline CÓDIGO & Participante & $\mathbf{1}$ & $\mathbf{2}$ & $\mathbf{3}$ & $\mathbf{4}$ & $\mathbf{5}$ & $\mathbf{6}$ & $\mathbf{7}$ & Tendencias \\
P4 & Andrea & 1 & 1 & 1 & 1 & 1 & 1 & 0 & 6 \\
P1 & Araceli & 1 & 0 & 1 & 1 & 0 & 1 & 0 & 4 \\
P2 & María Paula & 1 & 0 & 1 & 1 & 0 & 1 & 0 & 4 \\
P3 & Arelis & 1 & 0 & 1 & 0 & 1 & 1 & 0 & 4 \\
P5 & Victoria & 0 & 0 & 1 & 1 & 1 & 1 & 0 & 4 \\
P9 & Amanda & 1 & 1 & 1 & 1 & 0 & 0 & 0 & 4 \\
P6 & Daniela & 1 & 1 & 0 & 0 & 1 & 0 & 0 & 3 \\
P10 & Jessica & 0 & 0 & 0 & 1 & 1 & 1 & 0 & 3 \\
P7 & Lorena & 1 & 0 & 0 & 0 & 1 & 0 & 0 & 2 \\
P8 & Diana & 0 & 0 & 1 & 1 & 0 & 0 & 0 & 2 \\
& & 7 & 3 & 7 & 7 & 6 & 6 & 0 & \\
\hline
\end{tabular}

Fuente: Elaborado por los autores

Las ponderaciones cumplieron con el patrón ideal en el primer bloque de afirmaciones; es decir, hubo un equilibro entre una postura flexible y una más crítica. En el segundo bloque, la mayoría muestra inconformidad ante la imagen de la mujer en la publicidad, lo cual indica que la mayoría de participantes está lista para recibir contenido publicitario en el que se refleje a la mujer de una forma distinta a la tradicional.

Otro dato que vale la pena mencionar es que en las respuestas se presenta un índice alto de inconsistencias. Las afirmaciones fueron diseñadas con el fin de mantener una misma variable replicada de diferentes formas en varios enunciados, por lo que, si las participantes respondieron favorablemente a una de las dos tendencias, se espera que se la mantenga a lo largo de todas las afirmaciones; sin embargo, la mayoría tuvo respuestas aleatorias. La respuesta a esto puede ser la falta de compresión en los enunciados o por la asignación de respuestas al azar. 
A través del cuestionario de afirmaciones, también se puede ampliar las conclusiones y señalar que las actitudes de las participantes frente a la publicidad, en general, son positivas; es decir, se encuentran receptivas a los mensajes publicitarios.

Tabla 5 Resultados del cuestionario de actitudes hacia la representación de la mujer en la publicidad

\section{ACTITUDES FRENTE A LA MUJER EN LA PUBLICIDAD}

\begin{tabular}{c|ccccccccccccc}
\hline CÓDIGO & Participantes & $\mathbf{1}$ & $\mathbf{2}$ & $\mathbf{3}$ & $\mathbf{4}$ & $\mathbf{5}$ & $\mathbf{6}$ & $\mathbf{7}$ & $\mathbf{8}$ & $\mathbf{9}$ & $\mathbf{1 0}$ & $\mathbf{1 1}$ \\
P6 & Daniela & 1 & 0 & 1 & 1 & 0 & 1 & 1 & 1 & 0 & 0 & 1 & 7 \\
P2 & María Paula & 1 & 0 & 1 & 0 & 1 & 1 & 1 & 0 & 1 & 0 & 0 & 6 \\
P3 & Arelis & 1 & 0 & 1 & 0 & 0 & 0 & 1 & 1 & 1 & 1 & 0 & 6 \\
P4 & Andrea & 0 & 0 & 0 & 0 & 1 & 0 & 1 & 1 & 0 & 1 & 1 & 5 \\
P5 & Victoria & 1 & 0 & 0 & 0 & 0 & 0 & 1 & 0 & 0 & 1 & 1 & 4 \\
P1 & Araceli & 1 & 0 & 0 & 0 & 0 & 1 & 0 & 0 & 0 & 0 & 1 & 3 \\
P7 & Lorena & 0 & 0 & 0 & 1 & 0 & 0 & 0 & 1 & 0 & 0 & 1 & 3 \\
P9 & Amanda & 0 & 0 & 0 & 0 & 0 & 0 & 1 & 1 & 0 & 0 & 0 & 2 \\
P10 & Jessica & 0 & 0 & 0 & 1 & 0 & 0 & 0 & 1 & 0 & 0 & 0 & 2 \\
P8 & Diana & 0 & 0 & 0 & 0 & 0 & 0 & 0 & 1 & 0 & 0 & 0 & 1 \\
& & 5 & 0 & 3 & 3 & 2 & 3 & 6 & 7 & 2 & 3 & 5
\end{tabular}

En cuanto a las afirmaciones sobre la imagen de la mujer en la publicidad, se puede concluir que las participantes reconocer que la representación de la mujer es sexista, exagerada y poco realista, pero esta molestia no llega a influir en sus decisiones de compra. Con el grupo focal se pudo entender que esto sucede porque están habituadas a la comunicación publicitaria tradicional, lo cual genera que la tergiversación de la mujer en estos medios pase desapercibida; sin embargo, tras la sesión del grupo focal, las participantes se vuelven más críticas frente a este hecho.

\section{Discusión}

Aunque a primera impresión las personas sujetas de estudio denotaron expresiones de identificación y agrado hacia los spots presentados y su temática, a medida que se desarrollaba el ejercicio de investigación sus opiniones evolucionaron y la mayoría aseguró que más que una publicidad enfocada en la mujer, les gustaría ver anuncios en los que exista equidad de género y se muestre a hombres y mujeres compartiendo en igualdad de condiciones. Esta postura fue desencadenada cuando se comentó a las participantes que el femvertising también era denominado como "publicidad feminista". Sus principales argumentos se sustentaron en el spot de Dove y en el de Nosotras Ecuador, ya que en ambos se focalizaba en la mujer una problemática que afectaba a ambos 
géneros. Mientas que con el spot de Nike su actitud continuaba siendo de apoyo y validación al spot, puesto que la calidad de la composición semántica y audiovisual no daba pie para emitir comentarios en contra, ya que se enfocaban en actualizar los estereotipos conservadores de la mujer y no en compararla con el género masculino o en exponer sus defectos.

Es importante recalcar que esta postura fue el resultado de un método de investigación; es decir, las participantes llegaron a las conclusiones mencionadas porque la mediadora generaba preguntas que incitaban al debate y a la reflexión. Seguramente, si las mismas mujeres hubiesen estado en otro contexto o se hubiese detenido el ejercicio después de sus primeros comentarios, su actitud de apoyo hacia la tendencia no se habría modificado.

En este sentido, la investigación también arrojó que, pese a que Nike tuvo mayor apoyo y menos críticas que Dove, este último es el que terminó teniendo mayor recordación; la razón se debe a que todo contenido que incite a un proceso de reflexión o interiorización va a permanecer por más tiempo y, muy probablemente, cada vez que el perceptor reciba un mensaje de la marca, recordará la temática del spot. En el caso concreto de la temática de Dove, las participantes reflexionaron sobre la influencia de sus madres sobre ellas y la de ellas cuando llegue su turno de ser mamás. Más que defender una causa, les dejó una lección de vida; algo que ninguno de los otros spots analizados generó.

Como cualquier otro producto audiovisual, para garantizar la efectividad del femvertising no solo se debe prestar atención a las situaciones o escenas que empoderen o reivindiquen a la mujer, sino también se debe cuidar de la calidad y composición de las escenas, una duración adecuada para la publicidad en medios digitales - no más de 1:30, la concreción del mensaje y la coherencia entre el producto y la causa. Esto se debe a que las personas y, generalmente, las mujeres tienden a ser más detallistas con: la música, elementos visuales, voces, texto y a elementos narrativos del storytelling que ofrecen una experiencia más significativa. De igual manera, el ser humano recuerda más y mejor lo que recibe por el nervio óptico que lo que recibe por el nervio auditivo.

Respecto a la percepción de la mujer en el femvertising, aplauden a aquellos anuncios que las muestran como fuertes, competentes, decididas y con carácter. Mientras que rechazan aquellos en los que se las muestra como víctimas, débiles, con necesidad de protagonismo o con baja autoestima. Sin duda, ellas prefieren spots con un tono positivo y motivacional, más que los comerciales en los que se enfocan en los defectos o las situaciones negativas por las que atraviesan algunas mujeres. 


\section{Bibliografía}

Abuín, N. (2009). Publicidad, Roles Sociales Y Discurso De Género. Actes de Congènere, 25, 12.

Angulo Moncayo, Natalia Alexandra; Estrella Osorio, Arturo Virgilio y López Paredes, Marco (2018). La política en Twitter. Un estudio comparativo de las estrategias dis-cursivas de los candidatos finalistas a la Presidencia de Ecuador en 2017. En: adCo-munica. Revista Científica de Estrategias, Tendencias e Innovación en Comunicación, $\mathrm{n}^{\mathrm{o}} 16$. Castellón: Asociación para el Desarrollo de la Comunicación adComunica y Universitat Jaume I, 25-44. DOI: http://dx.doi.org/10.6035/2174-0992.2018.16.3

Bassat, L. (2017). El libro rojo de la publicidad: (Ideas que mueven montañas) (Séptima). España: Penguin Random House.

Becker-Herby, E. (2016). The Rise of Femvertising: Authentically Reaching Female Consumers. University of Minnesota. Recuperado de https://conservancy.umn.edu/bitstream/handle/11299/181494/BeckerHerby_\%20Final\%20Capstone_2016.pdf;sequence=1

Bourhis, R. Y., Gagnon, A., \& Moïse, L. C. (1996). Discriminación y relaciones intergrupales. En Estereotipos, discriminación y relaciones entre grupos, 1996, ISBN 84-481-0694-6, págs. 139-169 (pp. 139-169). McGraw-Hill Interamericana de España. Recuperado de https://dialnet.unirioja.es/servlet/articulo? codigo $=989066$

Canto, L. F. del. (2017, marzo 7). Perspectiva de género y cuenta de resultados. $\begin{array}{llllll}\text { Recuperado } & 19 \text { de } & \text { junio de }\end{array}$ https://cincodias.elpais.com/cincodias/2017/03/07/empresas/1488903754_69547 8.html

Catterall, M., Maclaran, P., \& Stevens, L. (1997). Marketing and feminism: a bibliography and suggestions for further research. Marketing Intelligence \& Planning, 15(7), 369-376. https://doi.org/10.1108/02634509710193208

Cruz Souza, F. (2011). Género, psicología y desarrollo rural : la construcción de nuevas identidades: las representaciones sociales de las mujeres en el medio rural. España: Ministerio de Agricultura, Pesca y Alimentación. Recuperado de http://www.mapama.gob.es/ministerio/pags/biblioteca/fondo/87506.htm

Dove. (2018). Real Beuty. Recuperado 19 de junio de 2018, de http://www.dove.com/es/home.html

Fair, H. (2011). La función de los medios masivos de comunicación en la legitimación de las reformas de mercado: Consideraciones a partir del caso argentino durante el primer gobierno de Carlos Menem (1989-1995). Revista SAAP, 5(1), 93-130. 
García Canclini, N. (2001). Culturas híbridas: estrategias para entrar y salir de la modernidad. México: Grijalbo [u.a.].

García, J. (2015). Publicidad, comunicación y cultura. Perspectivas teóricas para el estudio de la publicidad. España: UOC. Recuperado de https://www.unebook.es/es/libro/publicidad-comunicacion-y-cultura_46574

González, B. G. (1999). Los estereotipos como factor de socialización en el género. Comunicar, (12). Recuperado de http://www.redalyc.org/articulo.oa?id=15801212

Kotler, P., \& Armstrong, G. (1996). Fundamentos de Marketing (6ta ed.). México: Prentice Hall.

Krolokke, C., \& Sorensen, A. S. (2005). Gender Communication Theories and Analyses: From Silence to Performance (1 edition). Thousand Oaks, Calif: SAGE Publications, Inc.

Mieiro, A. S. de P. (2015). Las actitudes de las consumidoras hacia el fenómeno "femvertising" : estudio comparativo de la respuesta de consumidoras de la Generación Y hacia la publicidad pro-mujer (Tesis de pregrado). Universidad Pontificia Comillas, Madrid, España. Recuperado de https://repositorio.comillas.edu/xmlui/handle/11531/4440

Naranjo, S. E. (2016). La equidad de marca «brand equity» una estrategia para crear y agregar valor. Estudios Gerenciales, 35-42.

Niño, V. (1996). Los procesos de la comunicación y del lenguaje: fundamentos y práctica. Bogotá, Colombia: ECOE.

Ojeda, E. C. (2016). FEMVERTISING: Publicidad con enfoque de empoderamiento, 22.

Pérez, M. P. R., \& Almanzor, M. G. (2017). «Femvertising»: estrategias de empoderamiento femenino en la publicidad española. Investigaciones Feministas, 8(2), 337-351. https://doi.org/10.5209/INFE.54867

Rampton, M. (2015). Four Waves of Feminism. Recuperado 19 de junio de 2018, de https://www.pacificu.edu/about/media/four-waves-feminism

Sánchez Aranda, J. (2003). Framing the Woman"s Image in Advertising | Communication \& Society, 16(2), 67-92.

Sánchez, J. G., Terrats, J. R. A., \& Rosas, A. C. (2011). Guía técnica para la construcción de escalas de actitud, 13. 
SheKnows Media. (2014). SheKnows unveils results of its Fem-vertising survey. Recuperado 19 de junio de 2018, de http://www.sheknowsmedia.com/insights/femvertising

Tajfel, H. (1984). Grupos humanos y categorías sociales (Vol. 9). Barcelona, España: Quaderns de Psicología. Recuperado de https://dialnet.unirioja.es/servlet/articulo?codigo $=5036366$

Weibel, K. (1997). Mirror, Mirror. Images of Women Reflected in Popular Culture. Garden City.

Wojcicki, S. (2016). Susan Wojcicki on the Effectiveness of Empowering Ads on YouTube. Recuperado 19 de junio de 2018, de https://www.thinkwithgoogle.com/consumer-insights/youtube-empowering-adsengage/ 